\title{
Current trends and clinical applications of optical coherence tomography in orthodontics: A literature review
}

\section{Współczesne możliwości wykorzystania optycznej tomografii koherencyjnej w ortodoncji - przegląd piśmiennictwa}

\author{
Hemanth Tumkur Lakshmikantha ${ }^{1, B-D}$, Naresh Kumar Ravichandran ${ }^{2, B, C}$, Hyo-Sang Park ${ }^{1, A, D-F}$ \\ 1 Department of Orthodontics, School of Dentistry, Kyungpook National University, Daegu, Korea \\ ${ }^{2}$ School of Electronics Engineering, College of IT Engineering, Kyungpook National University, Daegu, Korea \\ A - research concept and design; $B$ - collection and/or assembly of data; $C$ - data analysis and interpretation; \\ $\mathrm{D}$ - writing the article; $\mathrm{E}$ - critical revision of the article; $\mathrm{F}$ - final approval of the article
}

Address for correspondence

Hyo-Sang Park

E-mail: parkhs@knu.ac.kr

Funding sources

None declared

Conflict of interest

None declared

Received on 0ctober 10, 2017

Reviewed on 0ctober 11, 2017

Accepted on 0ctober 24, 2017

DOI

10.17219/dmp/79134

Copyright

○ 2017 by Wroclaw Medical University

and Polish Dental Society

This is an article distributed under the terms of the

Creative Commons Attribution Non-Commercial License

(http://creativecommons.org/licenses/by-nc-nd/4.0/)

\begin{abstract}
This paper presents an overview of the current knowledge about non-invasive investigations using optical coherence tomography (OCT) - structural imaging of oral tissues and biomaterials applied in vivo and in vitro - employed in the field of orthodontics. Optical coherence tomography is an emerging technology for producing high-resolution cross-sectional imagery. OCT provides cross-sections of tissues in a non-contact and non-invasive manner. The device measures the time delay and the intensity of light scattered or reflected off of biological tissues, which results in tomographic imaging of their internal structure. This is achieved by scanning tissues at a low resolution.

This paper aims to describe the application of OCT in the field of orthodontics, through previous studies investigating the development and disorders of natural tooth hard tissues; the paper also describes OCT studies on dental demineralisation and dental biomaterial characterisation. We explain the working principles of OCT and mention different types of OCT systems in use. Comparisons between OCT and other commonly used orthodontic diagnostic aids are also made and the possible future implications of OCT in orthodontics is discussed.
\end{abstract}

Key words: optical coherence tomography, non-invasive investigation, orthodontics

Słowa kluczowe: optyczna tomografia koherencyjna, badania nieinwazyjne, ortodoncja 
As in every other medical and dental specialisation, accurate diagnostic imaging is a key factor for orthodontic diagnosis and treatment planning. In addition, it is an essential tool that allows an orthodontist to closely monitor treatment progress and outcome. In 1896, the first intraoral radiograph was taken; since then, two-dimensional (2D) images have been the staple of orthodontic imaging. In recent times, numerous technological breakthroughs like computed tomography are emerging as the new face of imaging techniques in orthodontics. Current imaging modalities have limited resolution. As a result, they are not effective at detecting minute details in tooth tissues; smaller lesions such as tiny pre-caries and cracks always go undiagnosed. Dental radiography, including computed tomography, has limited imaging contrast. The contrast in radiographs arises from attenuation variation between different tissue composites. The human tooth consisting of enamel, dentin, and pulp - does not exhibit good X-ray contrast. One of the initial indicators of dental decay is demineralisation of the enamel, and on radiographs its presentation might sometimes be overlooked by a professional. A thorough understanding of the tooth and its microstructures, the mineralisation process, and its dynamic mineral exchange processes can aid in gauging the health of the tooth. To study the microstructures of the tooth, studies using imaging modalities such as atomic force microscopy (AFM) and the transmission electron microscope have been used in previous studies. The latter modality needs to be performed ex vivo and cannot be used to assess healthy tissues or be performed in real time.

High-resolution and high-contrast images are some of the benefits that optical coherence tomography (OCT) imaging provides. Polarisation-sensitive OCT is a new improvement in OCT technology which provides better contrast when imaging tissues and their surrounding environment compared to the contrast of regular OCT imaging.

In 1991, Fujimoto et al. initially reported on the applications of optical coherence tomography (OCT). ${ }^{1}$ Since then, copious clinical studies in diverse fields of ophthalmology, ${ }^{2-4}$ dermatology, ${ }^{5,6}$ gastroenterology, ${ }^{7-9}$ and dentistry ${ }^{10,11}$ have used OCT. OCT is a non-invasive, non-radiative optical diagnostic apparatus dependent on the principles of interferometers. With the use of a low-coherence, broadband near-infrared light source, a spatial resolution of $\sim 20 \mu \mathrm{m}$ and real-time images become a reality. ${ }^{12,13}$ The initial applications of OCT were to visualise the human retina and atherosclerotic plaque. ${ }^{1,14}$ This optical imaging approach facilitates the cross-sectional imaging of the microstructures of tissue in situ. Negating the need for excision and the processing of specimens, as in conventional biopsy and histopathology, is a fundamental advantage in using OCT. With the advent of improved optical specifications and system capabilities, OCT shows great potential in orthodontic research and clinical applications.
The latest developments in OCT technology have made it feasible to image non-transparent tissues, allowing the technology to be implemented in a wide range of medical specialisations. ${ }^{15-18}$ There are restrictions in imaging depth due to optical attenuation from tissue scattering and absorption. Nevertheless, images up to a depth of 2-3 cm can be achieved in most tissues, as with conventional biopsy and histology. Although ultrasound can reach deeper tissues, the resolution in OCT images is far superior to standard clinical imaging. The excellent resolution of OCT makes it a treasured ally to study in vitro arterial pathology and plaque morphologies. ${ }^{19}$

In recent times, many functional OCT systems have evolved, like the Doppler OCT (DOCT), ${ }^{20,21}$ polarisation-sensitive OCT (PS-OCT), ${ }^{22-24}$ endoscopic OCT, ${ }^{25,26}$ and acoustic OCT. ${ }^{27,28}$ These functional systems provide structural images in conjunction with unique optical characteristics such as tissue orientation and blood flow velocity.

The use of OCT has been gaining popularity in the field of dental research. In 1998 the first in vitro images of dental hard and soft tissues in a porcine model were released. ${ }^{29}$ Subsequently, the in vivo imaging of human dental tissue was developed. ${ }^{30}$ Multitudinous studies have been conducted using OCT in orthodontics and information regarding the enamel surface, microleakage around the bracket base, and enamel demineralisation in relation to the bracket base has been revealed with the use of OCT.

\section{Principles of OCT}

OCT functions similarly to the technique of ultrasound imaging, but it works with the principle of light scattering instead of sound. Two-dimensional cross-sectional images of the sample structure are generated by measuring the variation in path length differences of the backscattered light from the different layers of the sample structure. ${ }^{1}$ OCT uses the near-infrared region of the light spectrum as its source since infrared light has optimal penetration depth in biological tissue structures. The speed of light travelling through air is very fast, thus the backscattered signals cannot be measured directly and correlation or interferometry techniques are used for this purpose. The most widely used approach for measuring the time delay in backscattered light is with the low coherence interferometry technique. Earlier primary applications of low-coherence interferometry were utilised in the field of optics and optoelectronic devices. ${ }^{31,32}$ By using a known light path as a reference, we can measure the time delay or the path length difference between the backscattered light path reflected back from the sample and back from the known reference path. These measured values show the structural difference between the sample and the reference objects. The most commonly used interferometry technique for OCT is the Michelson interferometer. 
In a basic Michelson interferometer configuration, the light from a source is directed onto a prism or a beam splitter. Then, the two split beams of light are directed to a reference path with a known length and to the sample that is to be measured. The reference path is varied according to the sample structure so as to obtain a better visualisation of the image. The backscattered light from the sample and from the reference path is interfered using a prism or a beam splitter. The interfered light produces dark and bright fringes correlating to the sample structures and this final interference signal is detected by a photodetector at the interferometer output. The axial resolution in OCT is determined by the coherence length and the bandwidth of the light source. The lateral resolution is dependent on the wavelength used and the beam optics used. Hence, by using a broad-bandwidth source with low coherence and an appropriate beam optics setup, it is possible to achieve high-resolution cross-sectional images of the sample structure. Figure 1 is a graphical representation of the working principles of OCT.

\section{Types of optical coherence tomography}

Optical coherence tomography (OCT) can be broadly classified into time domain optical coherence tomography (TD-OCT) and Fourier domain optical coherence tomography (FD-OCT). TD-OCT produces tomographic images by moving the reference arm mirror a few millimetres in order to create interference for individual layers of the sample. This means the image acquisition time is relatively longer. With the introduction of the Fourier relation, depth scanning can be done without any moving parts in the reference setup. This was achieved by either encoding the optical frequency in time by using a spectrally scanned source, or by an array of detectors for parallel acquisition of all wavelengths of the broadband source utilised. This helped to achieve the real-time acquisition of OCT images at high speeds. With a hundredfold increase in the capture time, quicker three-dimensional imaging of samples became possible. The FD-OCT can be

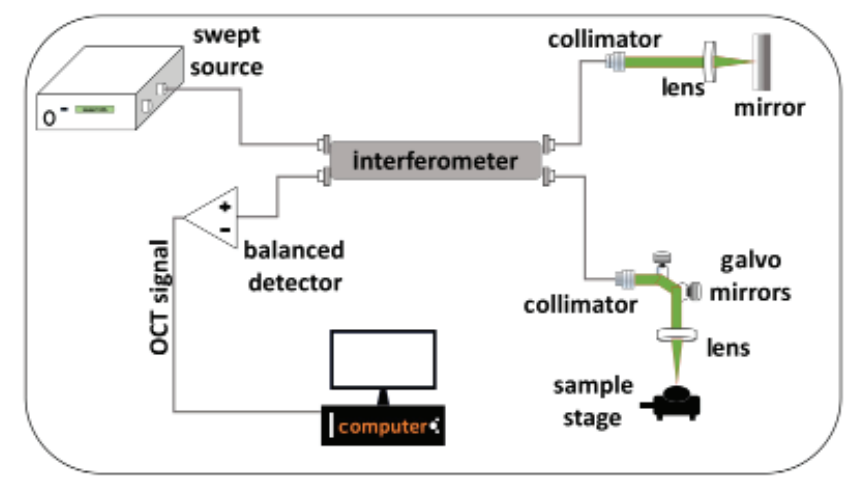

Fig. 1. Graphical representation of the working principle of optical coherence tomography further classified into 2 types depending on the type of source and detector used. The first is spectral domain optical coherence tomography (SD-OCT); the other method is swept source optical coherence tomography (SS-OCT). Both OCT types share a common configuration for the fixed reference arm and the sample arm. SD-OCT uses a broad-bandwidth source and the detector part is comprised of a combination of a dispersive medium (grating/ prism) followed by an array of detectors to simultaneously acquire the dispersed interference light. In the case of SS-OCT systems, the source bandwidth is spectrally scanned for individual wavelengths at high speed and the detector part is a single photodetector which individually acquires the interfered backscattered light signals one wavelength at a time. The SS-OCT has a higher depth sensitivity compared to SD-OCT but the acquisition time is slightly longer than for SD-OCT systems. By integrating the use of objective lenses and effectively utilising both coherence gating and confocal gating principles in OCT, it is possible to build optical coherence microscopy (OCM). By using high-magnification objective lenses and an expanded sampling beam waist, it is possible to attain a lateral resolution of $2 \mu \mathrm{m}$. The tradeoff is the limited depth of focus of OCM images.

\section{Comparison with other dental diagnostic methods}

Historically, newer imaging modalities replace existing technology to overcome their drawbacks. Computed tomography ushered in a new age of three-dimensional imaging with its appearance in the field of medical imaging, but its applications are limited due to its unnecessary exposure to ionising radiation and its inability to detect early lesions. Thus, the need arises to find a new, non-destructive technique that can be employed in the imaging of dental tissues. Currently, several innovative techniques are being developed for the diagnosis of dental diseases, such as a smart ultrasonic device, ${ }^{33-35}$ LED-based dental optical probes, ${ }^{36}$ and laser fluorescence..$^{37,38}$ Table 1 shows a comparison of dental OCT and other dental diagnostic modalities that are currently in practice. In short, OCT is a compelling diagnostic tool because it is a non-invasive, non-destructive, non-radiation-inducing, and real-time monitoring method with a high resolution and high contrast when compared to other imaging modalities.

\section{Use of OCT in orthodontics}

Formerly, the limitations of system size and light source manufacturing technology confined the applications of OCT in dentistry to studies involving visualisation of the morphology of dental hard and soft tissues. With the advent of well-engineered components, OCT applications 
Table 1. Comparisons between optical coherence tomography and other diagnostic methods used in dental research

\begin{tabular}{|c|c|c|}
\hline Methods & Advantages & Disadvantages \\
\hline Radiography & $\begin{array}{l}\text { low cost } \\
\text { broad measurement range }\end{array}$ & $\begin{array}{l}\text { radiative } \\
\text { poor spatial resolution } \\
\text { only } 2 \mathrm{D} \text { image }\end{array}$ \\
\hline Dental-CT & $\begin{array}{l}\text { broad measurement range } \\
3 \mathrm{D} \text { image reconstruction }\end{array}$ & $\begin{array}{l}\text { no real-time image } \\
\text { radiative } \\
\text { poor spatial resolution }\end{array}$ \\
\hline Intraoral Digital Cameras & $\begin{array}{l}\text { low cost } \\
\text { non-radiative }\end{array}$ & only surface information \\
\hline Periodontal probe & $\begin{array}{l}\text { low cost } \\
\text { broad measurement range }\end{array}$ & $\begin{array}{l}\text { low sensitivity } \\
\text { no image } \\
\text { invasive }\end{array}$ \\
\hline OCT & $\begin{array}{l}\text { high spatial resolution } \\
\text { real-time image } \\
\text { 3D image reconstruction is available }\end{array}$ & limited penetration depth and scanning range \\
\hline Raman spectroscopy & $\begin{array}{l}\text { high sensitivity } \\
\text { responses to mineral and chemical concentrations }\end{array}$ & $\begin{array}{l}\text { in vitro measurement } \\
\text { expensive } \\
\text { no image }\end{array}$ \\
\hline Laser fluorescence spectrometer & $\begin{array}{l}\text { real time detection } \\
\text { responses to bacteria and chemical concentrations }\end{array}$ & $\begin{array}{l}\text { lack of diagnostic consistency } \\
\text { no image }\end{array}$ \\
\hline
\end{tabular}

have increased and have been used to visualise dental and periodontal diseases. OCT has grown beyond its initial use as an imaging apparatus and with the onset of its real-time imaging and its inherent high-resolution capabilities, it has kicked off a plethora of orthodontic research opportunities.

In the field of orthodontics, a number of studies have been done to evaluate the enamel surface in relation to bonding and de-bonding procedures. Filho et al., in 2013, evaluated the enamel surface after de-bonding with metal and ceramic brackets. ${ }^{39}$ They studied the enamel fractures, adhesive remnants, and bracket fragments on enamel after de-bonding using OCT. In their study, the researchers were able to evaluate the damage caused to the enamel surface by different bracket systems and debonding procedures as well as to visualise and measure the adhesive remnant layer depth with the use of OCT.

Similarly, in 2015, Filho et al. studied the potential of OCT in evaluating the damage done to enamel by de-bonding and clean-up procedures by the use of metal and ceramic brackets. ${ }^{40}$ With the help of OCT, the researchers evaluated the damage done to the enamel and remnants of fractured ceramic brackets during de-bonding procedures, along with the adhesive and bracket mesh remnants on the surface of the enamel.

Seeliger et al. ${ }^{41}$ and Koprowski et al..$^{42}$ studied enamel thickness before and after orthodontic treatment using OCT and concluded that optical coherence tomography is an efficient aid in evaluating the surface and cross-sectional characteristics of enamel post-orthodontic treatment and it provides valuable information for deciding on the methodology and course of orthodontic treatment.

Nee et al. studied the use of cross-polarisation optical coherence tomography (CP-OCT) to longitudinally monitor demineralisation peripheral to orthodontic brack- ets. ${ }^{43}$ The study was conducted over a 12 -month period. Equipped with a high-speed CP-OCT the researchers extracted 3D images of the orthodontic bracket base. The images were analysed at intervals of 3 months in order to check for areas of demineralisation and remineralisation, and they also evaluated the use of a fluoride-releasing cement to check for remineralisation. They conferred the presence of and growth in the amount of demineralisation over time with the use of CP-OCT imaging. Additionally, the use of fluoride-releasing glass ionomer cement and conventional composite made no difference in the remineralisation of enamel around the bracket base.

Orthodontic adhesive materials can fail due to inherent voids between them and the bracket base, which can lead to bond failure and can delay orthodontic treatment progress. Though OCT cannot be used to quantify the bond strengths of orthodontic attachments, it can be applied to visualise and quantify the micro-damage at the junction of the bracket base and adhesive material. In the study done by Pithon et al., the researchers evaluated the in vitro effects of applying varnish containing casein phosphopeptide and amorphous calcium phosphate in the prevention of carious lesions around orthodontic brackets. ${ }^{45}$ Equipped with OCT imaging, the researchers were able to evaluate the depth of caries penetration beneath the applied dental varnish. They reported that the use of CPP-ACP reigned superior to conventional varnish in the prevention of white spot lesions around the bracket base. The use of OCT, with its high resolution, allowed the researchers to analyse the microstructures of the enamel with high degrees of definition.

The occurrence and progression of periodontal disease play a crucial role in the success of orthodontic treatment. Studies have shown that advancement of periodontal disease can lead to orthodontic treatment failure, making 
it vital for clinicians to observe, study, and monitor its progression. Many diagnostic modalities are available to clinically diagnose periodontal diseases. Recently, OCT has started to be used for that specific purpose. OCT renders high-resolution images that can be consistent and reproducible. With OCT the surface topography, pocket depths, their morphology, and attachment levels can be evaluated. ${ }^{44}$ The progression of periodontal disease, including gingival morphology, the characterisation of the cementum surface, and the presence and propagation of sub-gingival calculus is possible in great detail with OCT imaging. ${ }^{13}$ Colston et al. studied and evaluated OCT system efficiency to quantify gingival thickness along with alveolar crest morphology. ${ }^{29}$ Being able to visualise the structures in detail and with precision greatly contributes to formulating a periodontal surgical treatment plan.

Marcauteanu et al. used optical coherence tomography to study the microstructural characterisation of the temporomandibular disc. ${ }^{46}$ Temporomandibular joint discs were harvested from dead subjects and used in the study. Two different OCT systems were used. They compared the effectiveness of 2 OCT systems, one working at $1300 \mathrm{~nm}$ (time domain optical coherence tomography [TD-OCT]) and another at $840 \mathrm{~nm}$ (Fourier domain optical coherence tomography [FD-OCT]). The researchers scanned the harvested discs and evaluated them using the different OCT systems. Their results confirmed the OCT imaging technique to be the best for visualising the microstructural details of TMJ discs. They defined TD-OCT, due to its longer wavelength, to be particularly useful in the study of the homogenous microstructure of the thicker posterior pars. On the other hand, use of FD-OCT was faster. The researchers also advised that future studies with OCT be associated with arthroscopy, as OCT can be used to visualise the temporomandibular joint non-invasively and up to a sufficient depth.

Baek et al. determined the efficacy of OCT systems in determining tooth movement under light orthodontic forces ${ }^{47}$ Equipped with a TD-OCT, the researchers went about inducing orthodontic tooth movement in white rats. Post-induction of light orthodontic forces, structural
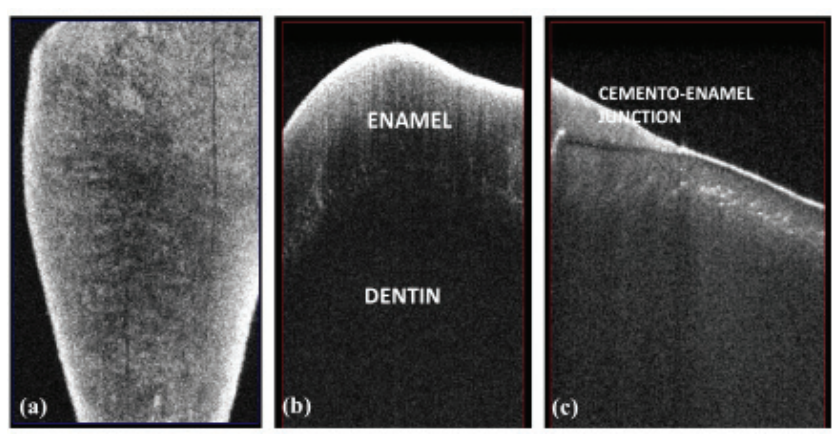

Fig. 2. OCT images of normal tooth hard tissues, from the orthodontic research archives. An image showing the tooth surface characterisation in different views, i.e., (a) the enamel surface, (b) a longitudinal section showing the enamel and dentinal layers of the tooth, and (c) the cementum - enamel junction of the tooth

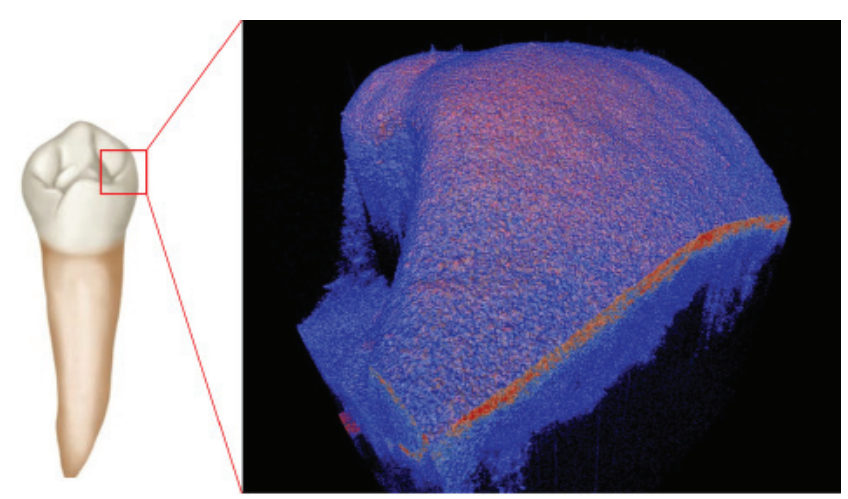

Fig. 3. 3D reconstruction of a premolar tooth, from the orthodontic research archives. The image depicts the 3D reconstruction of the scanned area using volume rendering software

variations in the periodontal ligament was studied using a digital radiography and TD-OCT system. With the use of OCT, they were able to successfully visualise the variations in the periodontal ligaments around the tooth, at different applications of force. In contrast, X-ray radiography could only confirm the presence of the PDL space and no discrimination of the variations within the PDL space as a reaction to orthodontic forces was possible. They concluded by suggesting that OCT can be used to predict tooth movement and can also be used to minimise the side effects associated with tooth movement by early detection.

Rashidifard et al. examined the ability of OCT, focusing primarily on structural OCT and PS-OCT, to identify and track early disease progression and its potential for the early diagnosis of osteoarthritis, rheumatoid arthritis, and rotator cuff repair. ${ }^{48}$ This preliminary study was done to show that OCT technology can be used to visualise inflammatory arthritis. Study of the inflammatory markers is vital to understanding the disease as a whole and to aid in the future management of the disease. Here the high resolution of OCT can help assess musculoskeletal diseases at micron-scale resolutions.

Figures 2 and 3 are from the orthodontic research archives of the Department of Orthodontics, Kyungpook National University. Figure 2 shows the enamel surface characterisation in different views, i.e., (a) the enamel surface, (b) a longitudinal section showing the enamel and dentinal layers of the tooth, and (c) the cementum-enamel junction of the tooth are seen. Figure 3 shows the $3 \mathrm{D}$ reconstruction of the scanned area using volume rendering software.

\section{Summary}

OCT is an effective new imaging technique for non-invasive in vivo and in vitro investigations in the field of orthodontics. In orthodontics, OCT provides a non-invasive, non-radiative, high-resolution image that can be utilised in a broad spectrum of clinical studies, ranging from studies involving hard and soft tissues to the various biomaterials used. 
For hard tissue imaging, OCT provides images in situ and in real time. Exposure to ionising radiation is a concern in modern-day clinical practice. OCT is an appropriate tool in this sense, as it is a non-invasive imaging modality that can provide images at a superficial level and also at considerable depth with high resolution and contrast for visualisation and analysis of dental hard tissue structures. OCT imaging allows early detection of demineralisation, which is commonly associated with the use of orthodontic brackets. A detailed visual analysis of remnants on the enamel surface after the de-bonding of orthodontic brackets can be created using OCT. The efficacy of various biomaterials that can influence the process of demineralisation can be visualised and analysed accurately with the help of OCT.

OCT allows for soft-tissue imaging, which is important in the treatment of periodontal diseases yet inaccessible to direct clinical assessment, and it offers great prospects for clinical studies involving tooth movement within the periodontal ligament. With the recent advances in OCT technology, it can be used to assess soft tissue lesions at a micron-scale resolution. The resolution might not be adequate for the study of single cells, but the architecture of the lesions can be studied. The measurements have no side effects and can be performed in real time.

Compared to other diagnostic methods used in orthodontics, the main advantage of using OCT lies in its higher resolution; OCT images display more detail and contrast than computed tomography and conventional radiography. There is the added advantage of being non-radiative. OCT imaging is also non-invasive, compared to other imaging techniques such as AFM and TEM, in which studies can only be performed ex vivo. Furthermore, the techniques are much simpler to perform than an MRI scan and much more detailed than ultrasound scans.

With the current system of OCT a number of images need to be analysed for investigation of an entire structure. In future, OCT systems with a higher depth penetration and with scanning heads purpose-built for orthodontic research can be made available. Various factors, including the components of the oral cavity and the degree of mineralisation of the teeth, impair the scattering and absorption of the light source; future developments may include an energy source of different wavelengths to target the specific components needed to investigate or to rule out the surrounding environmental noise. The entire process of scanning and acquisitioning the image is time-consuming, so developments in this area may see an even bigger jump in OCT in orthodontic research. Through the use of the diverse OCT techniques, for instance, polarisation-sensitive OCT, Doppler OCT, endoscopic OCT, optical coherence microscopy, and dual beam OCT, further research and advances in practical applications in the field of orthodontics need to be explored. By making the current OCT systems more robust and with scanning heads that are clinically applicable for orthodontic diagnosis, OCT can be utilised to its full potential.
OCT provides tissue sections in a non-contact and non-invasive manner and allows for real-time tissue imaging in situ, forgoing the need for biopsy, histological procedures, or the use of X-rays, so after solving the problems related to the availability and quality of equipment, it will be the method of choice in modern dental diagnostics. Increasing the number of clinical trials and the supplementary usage of OCT as an adjuvant in various studies is vital for OCT's growth in routine clinical practice and orthodontic research.

\section{References}

1. Huang D, Swanson EA, Lin CP, et al. Optical coherence tomography. Sci (New York, NY). 1991;254(5035):1178.

2. Wang Y, Bower BA, Izatt JA, Tan O, Huang D. Retinal blood flow measurement by circumpapillary Fourier domain Doppler optical coherence tomography. J Biomed Opt. 2008;13:64003.

3. Hangai M, Ojima $Y$, Gotoh $N$, et al. Three-dimensional imaging of macular holes with high-speed optical coherence tomography. Ophthalmol. 2007;114:763-773.

4. Yasuno $Y$, Hong $Y$, Makita $S$, et al. In vivo high-contrast imaging of deep posterior eye by $1-\mu \mathrm{m}$ swept source optical coherence tomography and scattering optical coherence angiography. Opt Express. 2007;15:6121-6139.

5. Pagnoni A, Knuettel A, Welker P, et al. Optical coherence tomography in dermatology. Ski Res Technol. 1999;5:83-87.

6. Pierce MC, Strasswimmer J, Park BH, Cense B, de Boer JF. Birefringence measurements in human skin using polarization-sensitive optical coherence tomography. J Biomed Opt. 2004;9:287-291.

7. Poneros JM, Brand S, Bouma BE, Tearney GJ, Compton CC, Nishioka NS. Diagnosis of specialized intestinal metaplasia by optical coherence tomography. Gastroenterol. 2001;120:7-12.

8. Evans JA, Poneros JM, Bouma BE, et al. Optical coherence tomography to identify intramucosal carcinoma and high-grade dysplasia in Barrett's esophagus. Clin Gastroenterol Hepatol. 2006;4:38-43.

9. Wilder-Smith P, Lee K, Guo S, et al. In vivo diagnosis of oral dysplasia and malignancy using optical coherence tomography: Preliminary studies in 50 patients. Lasers Surg Med. 2009;41:353-357.

10. Colston BW, Sathyam US, DaSilva LB, Everett MJ, Stroeve P, Otis LL. Dental oct. Opt Express. 1998;3:230-238.

11. Baumgartner A, Dichtl S, Hitzenberger CK, et al. Polarization-sensitive optical coherence tomography of dental structures. Caries Res. 2000;34:59-69.

12. Wojtkowski M, Srinivasan V, Fujimoto JG, et al. Three-dimensional retinal imaging with high-speed ultrahigh-resolution optical coherence tomography. Ophthalmol. 2005;112:1734-1746.

13. Fujimoto JG. Optical coherence tomography for ultrahigh resolution in vivo imaging. Nat Biotechnol. 2003;21:1361.

14. Brezinski ME, Tearney GJ, Weissman NJ, et al. Assessing atherosclerotic plaque morphology: Comparison of optical coherence tomography and high frequency intravascular ultrasound. Heart. 1997;77:397-403.

15. Schmitt JM, Knuttel A, Yadlowsky M, Eckhaus MA. Optical-coherence tomography of a dense tissue: Statistics of attenuation and backscattering. Phys Med Biol. 1994;39:1705.

16. Schmitt JM, Yadlowsky MJ, Bonner RF. Subsurface imaging of living skin with optical coherence microscopy. Dermatol. 1995;191:93-98.

17. Fujimoto JG, Brezinski ME, Tearney GJ, et al. Optical biopsy and imaging using optical coherence tomography. Nat Med. 1995;1:970-972.

18. Brezinski ME, Tearney GJ, Bouma BE, et al. Optical coherence tomography for optical biopsy: Properties and demonstration of vascular pathology. SPIE milestone Ser. 2001;165:628-635.

19. Fujimoto JG, Pitris C, Boppart SA, Brezinski ME. Optical coherence tomography: An emerging technology for biomedical imaging and optical biopsy. Neoplasia. 2000;2:9-25.

20. Zvyagin AV, FitzGerald JB, Silva K, Sampson DD. Real-time detection technique for Doppler optical coherence tomography. Opt Lett. 2000;25:1645-1647. 
21. Yang VXD, Mao YX, Munce N, et al. Interstitial Doppler optical coherence tomography. Opt Lett. 2005;30:1791-1793.

22. De Boer JF, Milner TE, van Gemert MJC, Nelson JS. Two-dimensional birefringence imaging in biological tissue by polarization-sensitive optical coherence tomography. Opt Lett. 1997;22:934-936.

23. Yasuno Y, Makita S, Sutoh Y, Itoh M, Yatagai T. Birefringence imaging of human skin by polarization-sensitive spectral interferometric optical coherence tomography. Opt Lett. 2002;27:1803-1805.

24. Pircher M, Goetzinger E, Leitgeb R, Hitzenberger CK. Three dimensional polarization sensitive OCT of human skin in vivo. Opt Express. 2004; 12:3236-3244.

25. Pan Y, Xie H, Fedder GK. Endoscopic optical coherence tomography based on a microelectromechanical mirror. Opt Lett. 2001;26:1966-1968.

26. Herz PR, Chen Y, Aguirre AD, et al. Ultrahigh resolution optical biopsy with endoscopic optical coherence tomography. Opt Express. 2004;12:3532-3542.

27. Lesaffre M, Farahi S, Gross M, Delaye P, Boccara AC, Ramaz F. Acousto-optical coherence tomography using random phase jumps on ultrasound and light. Opt Express. 2009;17:18211-18218.

28. Lesaffre M, Farahi S, Boccara AC, Ramaz F, Gross M. Theoretical study of acousto-optical coherence tomography using random phase jumps on ultrasound and light. JOSA A. 2011;28:1436-1444.

29. Colston BW, Everett MJ, Da Silva LB, Otis LL, Stroeve P, Nathel H. Imaging of hard-and soft-tissue structure in the oral cavity by optical coherence tomography. Appl Opt. 1998;37:3582-3585.

30. Drexler W, Fujimoto JG. Optical Coherence Tomography: Technology and Applications. Springer; 2015.

31. Takada K, Yokohama I, Chida K, Noda J. New measurement system for fault location in optical waveguide devices based on an interferometric technique. Appl Opt. 1987;26:1603-1606.

32. Youngquist RC, Carr S, Davies DEN. Optical coherence-domain reflectometry: A new optical evaluation technique. Opt Lett. 1987;12:158-160.

33. Roberts-Harry EA, Clerehugh V. Subgingival calculus: Where are we now? A comparative review. J Dent. 2000;28:93-102.

34. Meissner G, Oehme B, Strackeljan J, Kocher T. Clinical subgingival calculus detection with a smart ultrasonic device: A pilot study. J Clin Periodontol. 2008;35:126-132.

35. Meissner G, Oehme B, Strackeljan J, Kocher T. A new system to detect residual subgingival calculus: In vitro detection limits. J Clin Periodontol. 2006;33:195-199.

36. Krause F, Braun A, Jepsen S, Frentzen M. Detection of subgingival calculus with a novel LED-based optical probe. J Periodontol. 2005;76:1202-1206.

37. Krause $F$, Braun A, Frentzen $M$. The possibility of detecting subgingival calculus by laser-fluorescence in vitro. Lasers Med Sci. 2003;18:32-35.

38. Thomas SS, Mohanty S, Jayanthi JL, Varughese JM, Balan A, Subhash N. Clinical trial for detection of dental caries using laserinduced fluorescence ratio reference standard. J Biomed Opt. 2010;15:27001.

39. Leão Filho JCB, Braz AKS, Araujo RE de, Tanaka OM, Pithon MM. Enamel quality after debonding: Evaluation by optical coherence tomography. Braz Dent J. 2015;26:384-389.

40. Leão Filho JCB, Braz AKS, de Souza TR, de Araujo RE, Pithon MM Tanaka OM. Optical coherence tomography for debonding evaluation: An in-vitro qualitative study. Am J Orthod Dentofac Orthop. 2013;143:61-68.

41. Seeliger J, Machoy M, Koprowski R, Safranow K, Gedrange T, Woźniak K. Enamel thickness before and after orthodontic treatment analysed in optical coherence tomography. Biomed Res Int. 2017;2017:8390575.

42. Koprowski R, Machoy M, Woźniak K, Wróbel Z. Automatic method of analysis of OCT images in the assessment of the tooth enamel surface after orthodontic treatment with fixed braces. Biomed Eng Online. 2014;13:48.

43. Nee A, Chan K, Kang H, Staninec M, Darling CL, Fried D. Longitudinal monitoring of demineralization peripheral to orthodontic brackets using cross polarization optical coherence tomography. J Dent. 2014;42:547-555.

44. Todea C, Negrutiu ML, Balabuc C, et al. Optical coherence tomography applications in dentistry. Timisoara Med J. 2010;60:5-17.
45. Pithon MM, Dos Santos MJ, Andrade CSS, et al. Effectiveness of varnish with CPP-ACP in prevention of caries lesions around orthodontic brackets: An OCT evaluation. Eur J Orthod. 2014;37:177-182.

46. Mărcăuţeanua C, Demjana E, Sinescub C, et al. Preliminary optical coherence tomography investigation of the temporo-mandibular joint disc. Proc. SPIE. 2010:75542,G-1.

47. Baek JH, Na J, Lee BH, Choi E, Son WS. Optical approach to the periodontal ligament under orthodontic tooth movement: A preliminary study with optical coherence tomography. Am J Orthod Dentofac Orthop. 2009;135:252-259.

48. Rashidifard C, Vercollone C, Martin S, Liu B, Brezinski ME. The application of optical coherence tomography in musculoskeletal disease. Arthritis. 2013;2013:563268. 